\title{
Phenethyl isothiocyanate inhibits growth of human chronic myeloid leukemia K562 cells via reactive oxygen species generation and caspases
}

\author{
YATING WANG $^{1}$, SIXI WEI $^{1,2}$, JISHI WANG $^{1,3}$, QIN FANG $^{4}$ and QIXIANG CHAI ${ }^{1}$ \\ Departments of ${ }^{1}$ Hematology and ${ }^{2}$ Clinical Biochemistry, First Affiliated Hospital of Guiyang Medical College; \\ ${ }^{3}$ Hematopoietic Stem Cell Transplantation Center of Guizhou Province; ${ }^{4}$ Department of Pharmacy, \\ First Affiliated Hospital of Guiyang Medical College, Guiyang, Guizhou 550004, P.R. China
}

Received August 30, 2013; Accepted March 19, 2014

DOI: $10.3892 / \mathrm{mmr} .2014 .2167$

\begin{abstract}
Phenethyl isothiocyanate (PEITC), a potential cancer chemopreventive constituent of cruciferous vegetables, including watercress, has been reported to inhibit cancer cell growth by arresting the cell cycle and inducing apoptosis in various human cancer cell models. However, the role of PEITC in the inhibition of human chronic myeloid leukemia (CML) K562 cell growth and its underlying mechanisms have yet to be elucidated. In the present study, PEITC was found to induce cell death through the induction of reactive oxygen species (ROS) stress and oxidative damage. Heme oxygenase-1 (HO-1), which participates in the development of numerous tumors and the sensitivity of these tumors to chemotherapeutic drugs, plays a protective role by modulating oxidative injury. Therefore, the present study assessed the inhibitory effect of PEITC on K562 cells and whether HO-1 facilitated cell apoptosis and ROS generation. PEITC was found to suppress cell growth and cause apoptosis by promoting Fas and Fas ligand expression, increasing ROS generation and by the successive release of cytochrome $c$ as well as the activation of caspase-9 and caspase-3. PEITC was also combined with the HO-1 inhibitor zinc protoporphyrin IX and the inducer hemin to assess whether HO-1 determines cell survival and ROS generation. The results of the present study suggest that PEITC may be a potential anti-tumor compound for CML therapy, and that HO-1 has a critical function in PEITC-induced apoptosis and ROS generation.
\end{abstract}

Correspondence to: Professor Jishi Wang, Department of Hematology, First Affiliated Hospital of Guiyang Medical College, 28 Guiyi Street, Guiyang, Guizhou 550004, P.R. China

E-mail: jishiwangcn@126.com

Key words: phenethyl isothiocyanate, K562, apoptosis, ROS, HO-1

\section{Introduction}

Chronic myeloid leukemia (CML) is a myeloproliferative disorder that is characterized by the unregulated growth of myeloid leukemia cells in the bone marrow and their accumulation in the blood. Epidemiologic data suggest that the rate of CML incidence is one to two per 100,000 individuals, and that $15-20 \%$ of adult leukemia cases are CML (1). Conventionally, CML is treated using interferon- $\alpha$ and cytotoxic agents; however, these have limited use in the clinic due to adverse side effects. Despite improved therapeutic effects, targeted agents are limited by the development of drug resistance.

An increased dietary intake of cruciferous vegetables is associated with a reduced incidence of cancer (2). Phenethyl isothiocyanate (PEITC) is an important compound obtained from cruciferous vegetables that is effective in numerous types of cancer, including prostate, breast, ovarian, oral and lung cancer (3-7). Several mechanisms, including the inhibition of cytochrome p450 enzymes, the induction of phase II detoxification enzymes (8) and the activation of death receptors 4 and 5 (9), are involved in the anti-tumor effects of PEITC. PEITC has also been shown to decrease the expression and function of the estrogen receptor (10). In addition, PEITC induces reactive oxygen species (ROS)-mediated cancer cell death by inhibiting oxidative phosphorylation (11). Therefore, PEITC induces cell death by causing ROS stress, which leads to oxidative damage. This can be prevented by antioxidants (12). However, the mechanism underlying the PEITC-induced apoptosis of human CML cells is yet to be elucidated.

Heat shock proteins (HSPs) are molecular chaperones that regulate protein function by participating in protein synthesis, accumulation, transport and degradation. The HSP heme oxygenase-1 (HO-1) is the rate-limiting enzyme in the heme degradation pathway (13) induced by inflammatory cytokines, oxidation, ischemia, hypoxia and endotoxins (14). HO-1 is a highly conserved stress protein that has been shown to act in a non-selective manner to confer protection to normal and cancerous organs, tissues and cells against harmful stimuli and pathological processes (15). The lack of selectivity of the protective function associated with HO-1 may result in tumor resistance to chemotherapeutic drugs (16). In a previous study, 
we demonstrated that $\mathrm{HO}-1$ can protect CML cells from apoptosis, and that an association exists between HO-1 and CML cell growth (17). The present study aimed to extend these findings and investigate the role of HO-1 in the regulation of PEITC-mediated apoptotic cell death. These molecules have been found to be critical regulators of apoptosis induced by various stimuli.

In the present study, the cytotoxic effect of PEITC on K562 cells was investigated in vitro. Furthermore, the effects of HO-1 on K562 cell survival and ROS generation were assessed using an HO-1 inhibitor, zinc protoporphyrin IX (ZnPPIX), and inducer, hemin. PEITC was found to induce cell death through the Fas/Fas ligand (FasL) pathway, ROS generation and caspase activation, and HO-1 was found to have a crucial role in PEITC-induced apoptosis and ROS generation.

\section{Materials and methods}

Cell culture. Human leukemia K562 cells were obtained from the Affiliated Hospital of Guiyang Medical University (Guiyang, China). Cells were maintained in RPMI-1640 medium containing $10 \%$ fetal bovine serum, $100 \mathrm{U} / \mathrm{ml}$ penicillin and $100 \mathrm{~g} / \mathrm{ml}$ streptomycin at $37^{\circ} \mathrm{C}$ in an incubator containing $5 \% \mathrm{CO}_{2}$ and humidified air. The study was approved by the ethics committee of Hospital affiliated to Guiyang medical college (Guiyang, China).

Cytotoxicity assays. Two different methods were used to evaluate the cytotoxic effects of PEITC under various experimental conditions. Cell viability was determined using the MTT assay. Briefly, cells were seeded at a density of 20,000 cells/well on 96-well plates, treated with various concentrations of PEITC and then cultured for 24, 48 and $72 \mathrm{~h}$ at $37^{\circ} \mathrm{C}$ with $5 \% \mathrm{CO}_{2}$. Following culture, the medium was replaced with $200 \mu \mathrm{l}$ fresh medium containing MTT at a final concentration of $250 \mu \mathrm{g} / \mathrm{ml}$. Subsequent to incubation at $37^{\circ} \mathrm{C}$ for $4 \mathrm{~h}$, the reaction was terminated with the addition of $150 \mu \mathrm{l}$ dimethyl sulfoxide (DMSO)/well. The solution was then agitated for $10 \mathrm{~min}$ to dissolve the crystals. The absorbance of each sample well was read at $492 \mathrm{~nm}$ using an automated 96-well plate reader. All assays were performed in triplicate. The inhibition ratio (I\%) was calculated using the following formula:

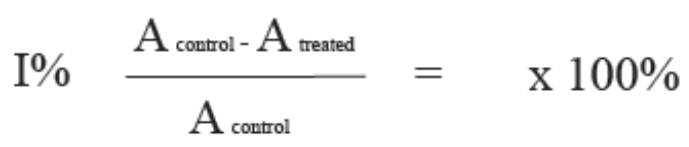

Cell death was determined using flow cytometric analysis, subsequent to the cells being double-stained with Annexin-V-fluorescein isothiocyanate (FITC) and propidium iodide (PI). Cells were incubated for $48 \mathrm{~h}$ following the addition of 5, 10, 15 and $20 \mathrm{~mol} / 1$ PEITC. The cells (10 $/$ sample) were then washed twice with cold phosphate-buffered saline (PBS) and suspended in $500 \mu 11 \mathrm{X}$ binding buffer (PBS solution included $10 \mathrm{mM}$ HEPES, $140 \mathrm{mM} \mathrm{NaCl}$ and $2.5 \mathrm{mM}$ $\mathrm{CaCl}_{2}, \mathrm{pH}$ 7.4). A total of $5 \mu \mathrm{l}$ PI was then added subsequent to the addition of $5 \mu \mathrm{l}$ Annexin-V-FITC. The reagents were mixed gently and the samples were incubated in the dark for $15 \mathrm{~min}$ at room temperature. The samples were then analyzed by flow cytometry using the BD FACSCalibur ${ }^{\mathrm{TM}}$ flow cytometer with BD CellQuest ${ }^{\mathrm{TM}}$ software (Becton, Dickinson and Company, Franklin Lakes, NJ USA).

Quantitative polymerase chain reaction ( $q P C R)$. Total RNA was extracted using TRIzol ${ }^{\circledR}$ Reagent (Invitrogen Life Technologies, Carlsbad, CA, USA). RNA was reverse transcribed into cDNA using a reverse transcription kit (Promega Corp., Madison, WI, USA), which was stored at $-20^{\circ} \mathrm{C}$. The primers (Takara Bio Inc., Dalian, China), shown in Table I, were designed using Primer Express 3.0 Software (Applied Biosystems, Foster City, CA, USA). qPCR was performed using a real-time PCR thermocycler (Applied Biosystems) and quantified using SYBR ${ }^{\circledR}$ Green PCR Master Mix (Applied Biosystems) using $1 \mu \mathrm{l}$ cDNA in a final reaction volume of $20 \mu \mathrm{l}$. The reactions were cycled 40 times subsequent to an initial denaturation step of $95^{\circ} \mathrm{C}$ for $10 \mathrm{~min}$. The PCR cycle conditions were as follows: Denaturation, $95^{\circ} \mathrm{C}$ for $15 \mathrm{sec}$; annealing, $60^{\circ} \mathrm{C}$ for $10 \mathrm{sec}$ and extension, $72^{\circ} \mathrm{C}$ for $10 \mathrm{sec}$. Following the PCR, the temperature was increased from 60 to $95^{\circ} \mathrm{C}$ at a rate of $2^{\circ} \mathrm{C} / \mathrm{min}$, and the fluorescence was measured every $15 \mathrm{sec}$ to construct the melting curve. GAPDH was used as an internal control. SDS 2.2.1 software (Applied Biosystems) was used to perform relative quantification of the target genes using the $\Delta \Delta \mathrm{Ct}$ method.

Western blot analysis. Cells were washed three times with ice-cold PBS and then lysed in Tris-buffered saline (TBS; $50 \mathrm{mmol} / 1$ Tris- $\mathrm{HCl}, \mathrm{pH} 8.0,150 \mathrm{mmol} / \mathrm{l} \mathrm{NaCl}, 100 \mu \mathrm{g} / \mathrm{ml}$ phenylmethylsulfonyl fluoride and $1 \%$ Triton $\mathrm{X}-100$ ) for $30 \mathrm{~min}$ on ice. Aliquots of cell lysates containing $20 \mu \mathrm{g}$ protein were separated using 12\% SDS-PAGE and transferred to nitrocellulose membranes. Subsequent to being blocked with 5\% skimmed milk in TBS, membranes were incubated with polyclonal antibodies against HO-1 (1:200), cytochrome $c$ (1:200), caspase-3 (1:100), caspase-8 (1:500), caspase-9 (1:500), Fas $(1: 1,000)$, FasL $(1: 1,000)$ and $\beta$-actin $(1: 500)$ overnight. The membranes were then washed in $0.1 \%$ Tween 20 in TBS and incubated with secondary antibodies (Cell signaling technology, Boston, MA, USA) at room temperature for $1 \mathrm{~h}$. Immunoreactive signals were visualized by exposing the membrane to Kodak X-ray film (Carestream Health, Inc., Xiamen, China), and quantified using Image J 1.44p (National Institutes of Health, Bethesda, MD, USA).

Intracellular ROS measurement. The measurement of intracellular ROS was based on the ROS-mediated conversion of non-fluorescent dichlorofluorescin diacetate (DCFH-DA) into DCFH. Briefly, K562 cells were treated by PEITC for an additional $48 \mathrm{~h}$. Cells were then centrifuged and resuspended in $1 \mathrm{X}$ PBS containing a final concentration of $10 \mu \mathrm{M}$ DCFH-DA and incubated at $37^{\circ} \mathrm{C}$ for $30 \mathrm{~min}$. Cells were subsequently washed with $1 \mathrm{X}$ PBS and analyzed using the FITC channel in a flow cytometer.

Statistical analysis. Data are expressed as the mean \pm standard deviation. Analysis of variance was performed using SPSS 13.0 (SPSS, Inc., Chicago, IL USA). A value of $\mathrm{P}<0.05$ was considered to indicate a statistically significant difference. 
Table I. Quantitative polymerase chain reaction primer sequences.

\begin{tabular}{llll}
\hline Gene & \multicolumn{1}{c}{ Forward primer } & \multicolumn{1}{c}{ Reverse primer } \\
\hline Fas & 5'-AGCTTGGTCTAGAGTGAAAA & 5'-GAGGCAGAATCATGAGATAT & 180 \\
Fas ligand & 5'-CACTTTGGGATTCTTTCCAT & 5'-GTGAGTTGAGAGCTACAGA & 160 \\
Cytochrome $c$ & 5'-GGGCGAGAGCTATCTAATGCAAG & 5'-TACAGCCAAAGCAGCAGCTCA \\
Caspase-3 & 5'-GACTCTGGAATATCCCTGGACAACA & 5'-AGGTTTGCTGCATCGACATCTG \\
Caspase-8 & 5'-CAAGAGGAAATCTCCAAATGCAAC & 5'-CAGGATGTCCAACTTTCCTTCTCC & 132 \\
Caspase-9 & 5'-GAGCAGTGGGCTCACTCTGAA & 5'-GGAAATTAAAGCAACCAGGCATC & 108 \\
GAPDH & 5'-GAAGGTGAAGGTCGGATGC & 5'-GAAGATGGTGATGGGATTTC & 226 \\
\hline
\end{tabular}
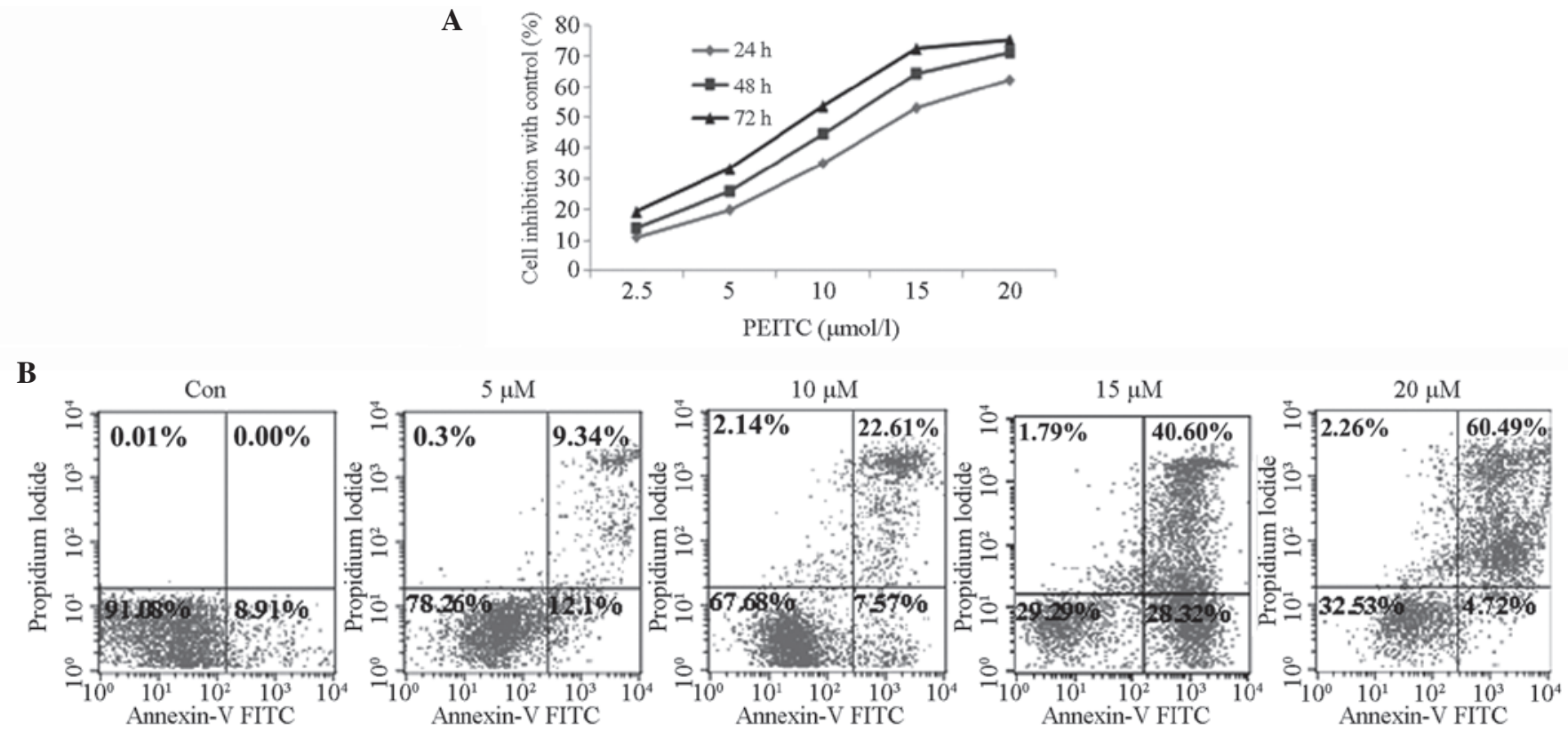

Figure 1. (A) Growth inhibition and viability of K562 cells treated with PEITC for 24,48 and $72 \mathrm{~h}$ at the indicated concentrations. Significant inhibitory effects with reduced viability were detected in the cells at the threshold of $10 \mu \mathrm{mol} / 1(\mathrm{P}<0.05)$. Results are presented as the mean \pm standard deviation from three independent experiments. (B) Subsequent to treatment with PEITC $(5,10,15$ and $20 \mu \mathrm{mol} / \mathrm{l})$ for $48 \mathrm{~h}$, cells were harvested and stained with Annexin V-FITC/PI. The cell apoptosis rate was determined using flow cytometry. Representative flow cytometry scattergrams of Annexin V-FITC/PI-stained cells revealed four cell populations. Early apoptosis was shown by positive Annexin V-FITC and negative PI staining of cells in the lower right section of the scattergram. Late apoptosis was shown by positive Annexin V-FITC and PI staining of cells in the upper right section. PEITC, phenethyl isothiocyanate; FITC, fluorescein isothiocyanate; PI, propidium iodide; Con, control.

\section{Results}

Effect of PEITC on K562 cell proliferation and apoptosis. The effect of treatment with various concentrations of PEITC $(0$, $2.5,5,10,15$ and $20 \mu \mathrm{mol} / \mathrm{l})$ for various time intervals $(24$, 48 and 72 h) on K562 cell growth was investigated. As shown in Fig. 1A, PEITC inhibited cell proliferation in a dose- and time-dependent manner. Therefore, the inhibition of K562 cell proliferation using high concentrations of PEITC for a long duration may be cytotoxic. The apoptosis-inducing effect of PEITC on K562 cells was determined using flow cytometry. Following treatment with 5, 10, 15 and $20 \mu \mathrm{mol} / 1$ PEITC for $48 \mathrm{~h}$, apoptosis was induced in $21.45 \pm 1.68,30.06 \pm 1.94$, $68.51 \pm 1.95$ and $66.09 \pm 2.01 \%$ of K562 cells, respectively (Fig. 1B).

qPCR analysis. To investigate the effect of PEITC on the expression of apoptosis-associated genes, K562 cells were exposed to $5,10,15$ and $20 \mu \mathrm{mol} / 1$ PEITC for $48 \mathrm{~h}$ and gene expression levels were analyzed using qPCR. As shown in Fig. 2, exposure to PEITC significantly increased the mRNA expression of cytochrome $c$, the key enzyme responsible for caspase-9 activation $(\mathrm{P}=0.024)$. The mRNA expression of the apoptosis-related genes caspase- 3 and -8, Fas and FasL was also observed to be increased by PEITC.

Western blot analysis. Following treatment with the aforementioned concentrations of PEITC for 48 h, K562 cell extracts were collected for western blot analysis. As shown in Fig. 3, PEITC treatment was found to increase the protein expression of cytochrome $c$, caspase-9, -3 and -8, Fas and FasL. These results are in accordance with those of the qPCR analysis.

PEITC elevates ROS production in K562 cells. To investigate whether the PEITC-induced cytotoxic effects in K562 cells involved ROS, ROS levels were measured using flow 


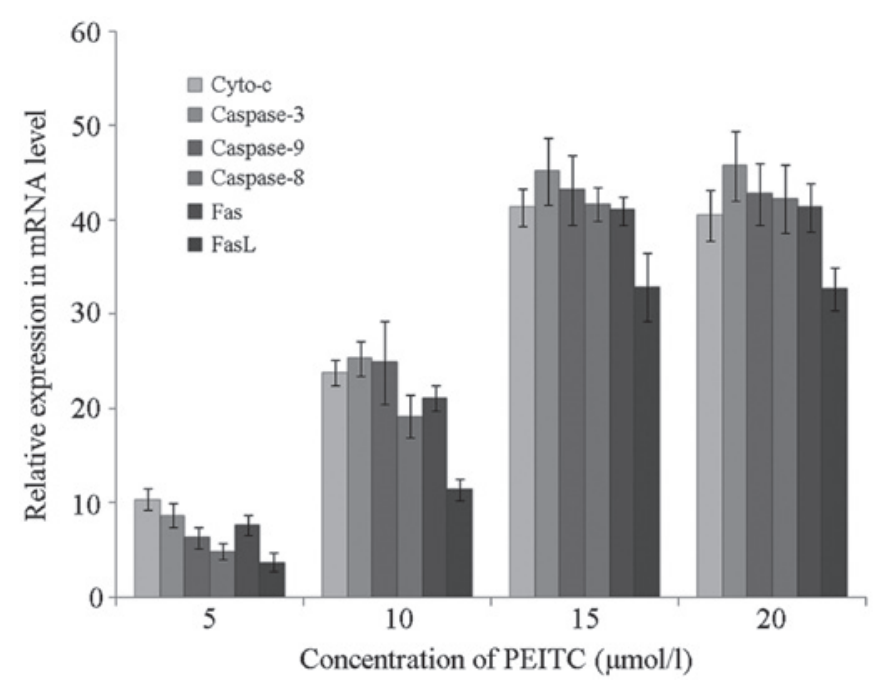

Figure 2. Cyto- $c$, caspase-3, -8 and -9 , Fas and FasL mRNA expression in K562 cells treated with PEITC, analyzed using quantitative polymerase chain reaction. Data were analyzed according to the $\Delta \mathrm{CT}$ method and are presented as the mean \pm standard deviation of three independent experiments. PEITC, phenethyl isothiocyanate; FasL, Fas ligand; CT, cycle threshold; Cyto- $c$, cytochrome $c$.

$\mathbf{A}$

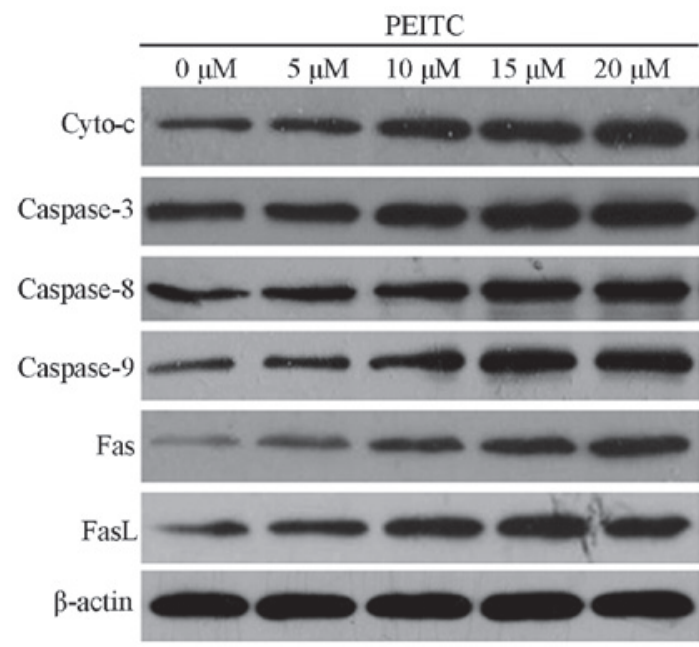

B

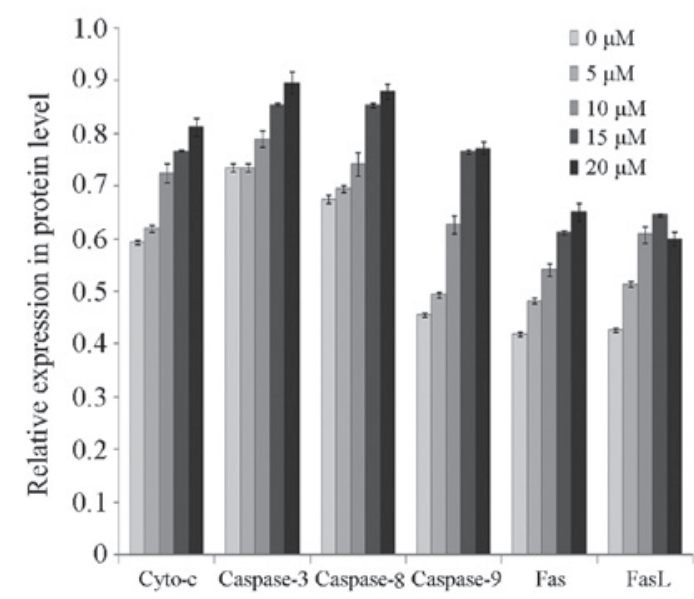

Figure 3. (A) K562 cells treated with PEITC (5, 10, 15 and $20 \mu \mathrm{M})$ for $48 \mathrm{~h}$. Cell lysates were subjected to western blot analysis with antibodies against cyto- $c$, caspase- $3,-9$ and -8 , Fas and FasL. $\beta$-actin was used as a loading control. Results are representative of two independent experiments. (B) Relative mean protein expression of apoptosis-associated genes normalized to $\beta$-actin expression. PEITC, phenethyl isothiocyanate; FasL, Fas ligand; Cyto- $c$, cytochrome $c$.
A

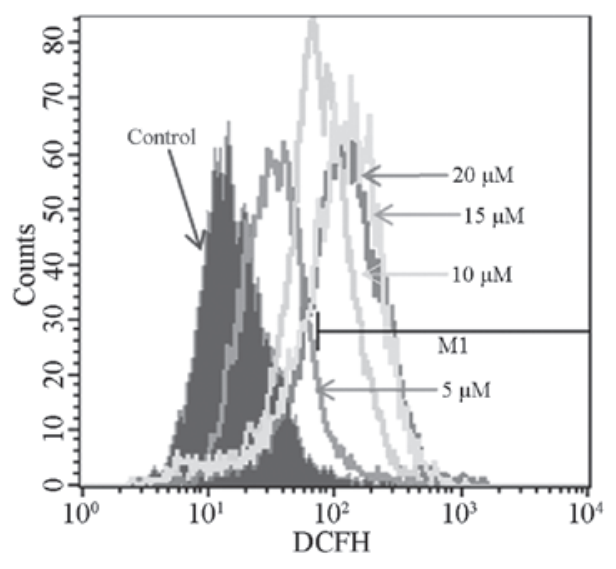

B

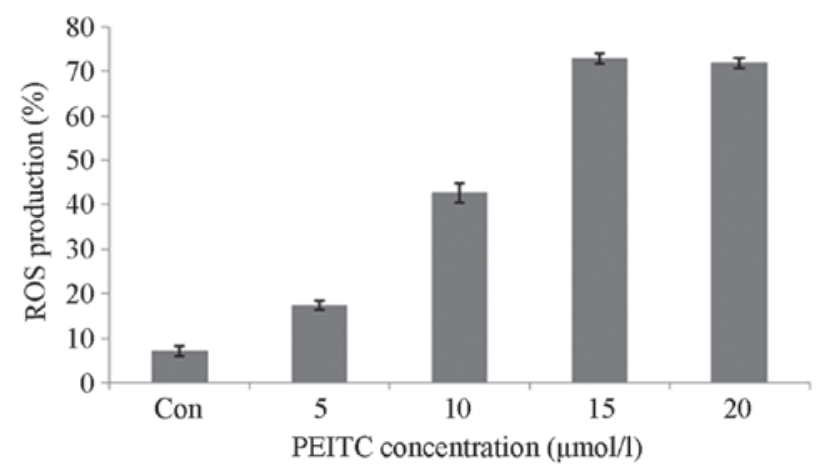

Figure 4. Figure 4. (A) PEITC affects ROS production in K562 cells treated with 5, 10,15 and $20 \mu \mathrm{mol} / 1$ PEITC for $48 \mathrm{~h}$. All samples were measured using flow cytometric analysis subsequent to being stained with DCFH-DA and dried. (B) ROS generation in K562 cells. Data are presented as the mean \pm standard deviation of three independent replicates. PEITC, phenethyl isothiocyanate; ROS, reactive oxygen species; DCFH-DA, 2,7-dichlorodihydrofluorescein diacetate; Con, control.

cytometry. As shown in Fig. 4, PEITC concentrations of 5, 10, 15 and $20 \mu \mathrm{mol} / 1$ were found to promote ROS production in K562 cells.

HO-1 protects $\mathrm{K} 562$ cells from PEITC-induced apoptosis. In the present study, PEITC was observed to induce apoptosis and elevate ROS production in K562 cells; therefore, the impact of HO-1 on cell survival was investigated. To assess the effect of HO-1 expression on PEITC-induced apoptosis, PEITC was combined with the HO-1 inhibitor ZnPPIX and the HO-1 inducer hemin in order to decrease and increase the expression of HO-1 in K562 cells, respectively. Cells were cultured with $20 \mu \mathrm{mol} / 1$ hemin and $10 \mu \mathrm{mol} / 1 \mathrm{ZnPPIX}$ for $48 \mathrm{~h}$, and the expression levels of the apoptosis-related genes cytochrome $c$, caspase- $9,-3$ and -8 , Fas and FasL were detected and compared with those of the cells treated solely with PEITC (Figs. 5 and 6). HO-1 was observed to protect K562 cells from PEITC-induced apoptosis.

Critical role of HO-1 in K562 cancer cell ROS generation. $\mathrm{HO}-1$ is involved in regulating the redox equilibrium in human cells; therefore, K562 cells were incubated with PEITC, hemin and PEITC plus hemin for $48 \mathrm{~h}$. As shown in Fig. 7, ROS production, which was facilitated by PEITC in a dose-dependent manner, was downregulated with high HO-1 expression. Furthermore, fluorescence-activated cell sorting analysis 


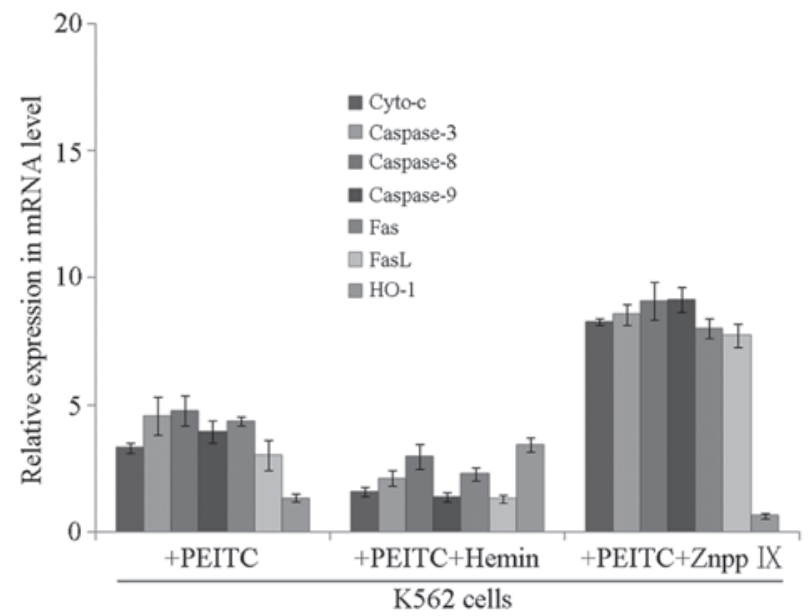

Figure 5. mRNA expression of the apoptosis-related genes cyto- $c$, caspase-3, -9 and -8 , Fas and FasL in the different treatment groups. Expression was highest in the PEITC + ZnPPIX treatment group and lowest in the PEITC + hemin treatment group. Data are presented as the mean \pm standard deviation of three independent experiments. PEITC, phenethyl isothiocyanate; FasL, Fas ligand; Cyto- $c$, cytochrome- $c$; HO-1, heme oxygenase-1; ZnppIX, zinc protoporphyrin IX.

A

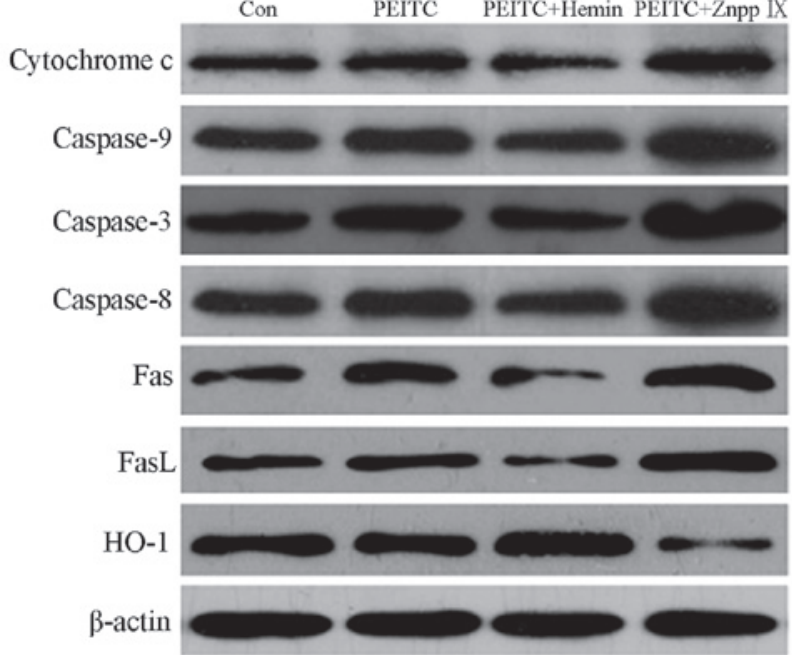

B

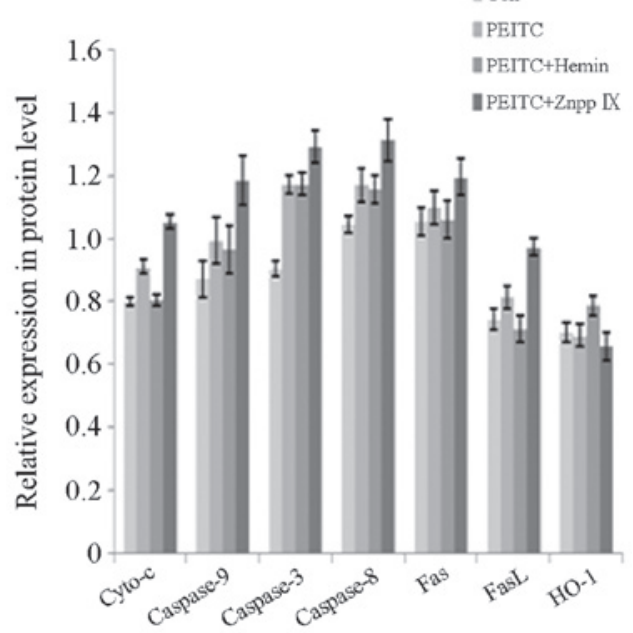

Figure 6. (A) Protein expression of the apoptosis-related genes cyto- $c$, caspase-3, -9 and -8, Fas and FasL in different treatment groups. Expression was highest in the PEITC + ZnPPIX treatment group and lowest in the PEITC + hemin group. (B) Mean protein expression of the apoptosis-related genes normalized to that of $\beta$-actin. Data are presented as the mean \pm standard deviation of three independent experiments. PEITC, phenethyl isothiocyanate; FasL, Fas ligand; Cyto- $c$, cytochrome- $c$; HO-1, heme oxygenase-1; ZnPPIX, zinc protoporphyrin IX.

A

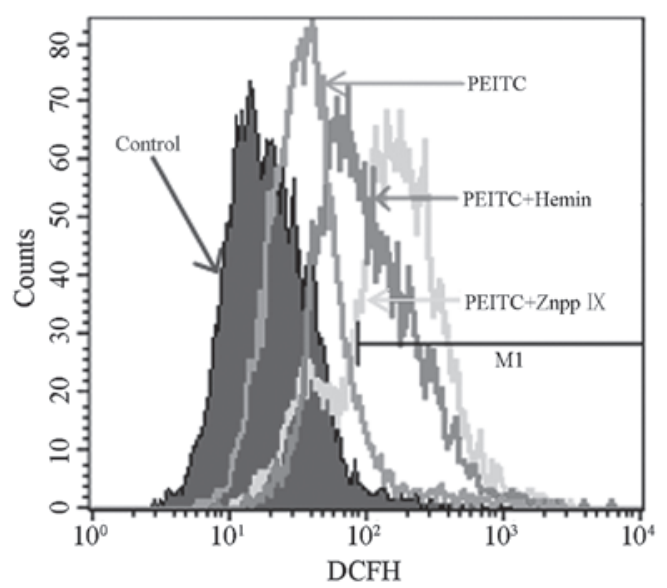

B

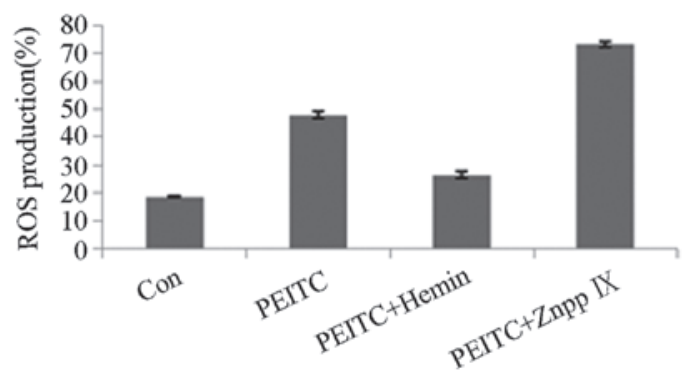

Figure 7. PEITC affects the levels of ROS production in K562 cells. (A) Fluorescence-activated cell sorting revealed that PEITC treatment significantly elevated ROS production in K562 cells $(\mathrm{P}<0.05)$, and that this elevation was attenuated upon treatment with hemin. This suggests that heme oxygenase-1 may be critical for ROS generation in K562 cells. (B) ROS generation in K562 cells. Data are presented as the mean \pm standard deviation of three independent replicates. PEITC, phenethyl isothiocyanate; ROS, reactive oxygen species; DCFH, 2,7-dichlorodihydrofluorescein; Con, control; ZnPPIX, zinc protoporphyrin IX. 
revealed that PEITC significantly elevated ROS production $(\mathrm{P}=0.017)$, and that this elevation was attenuated upon addition of hemin, indicating that the decreased ROS production originated from high HO-1 expression.

\section{Discussion}

PEITC is a potential cancer chemopreventive constituent of edible cruciferous vegetables and has been shown to inhibit various models of cancer, including prostate, breast, ovarian, oral and lung cancer (3-7). The present study aimed to characterize the anti-leukemia effects of PEITC. The PEITC-mediated inhibition of cancer development involves the induction of apoptosis; however, molecular regulators of this PEITC-mediated proapoptotic response remain undefined. The present study found that PEITC exhibited anti-tumor activity by suppressing K562 cell growth, inducing apoptosis and through mitochondria-dependent ROS generation.

Anti-cancer drugs are screened according to their cytotoxic effects on cell proliferation and viability. The results of the present study showed that PEITC reduced the percentage of viable K562 cells and induced apoptosis (Fig. 1). ROS are involved in cancer cell apoptosis (18). In the present study, flow cytometric analysis revealed that 5-20 $\mu \mathrm{mol} / 1$ PEITC promoted ROS generation in K562 cells (Fig. 4B). It has previously been shown that ROS have functions upstream of cytochrome $c$ release and caspase- 3 activation, which are induced by certain apoptotic stimuli, such as hyperoxia (19). These findings indicate that PEITC-induced apoptosis may also rely on the mitochondria. In the present study, PEITC was found to significantly increase the expression of caspase $-3,-8$ and -9 (Figs. 2 and 3), suggesting that PEITC may induce apoptosis through a caspase-dependent pathway. In summary, PEITC was found to promote apoptosis by activating caspase- $3,-8$ and -9 subsequent to inducing ROS production and cytochrome $c$ release through Fas and FasL.

PEITC has been suggested to induce several anti-inflammatory and anti-oxidative phase II/antioxidant enzymes (20), including HO-1. HO-1 is a highly conserved stress protein that has been shown to confer protection to organs, tissues and cells against harmful stimuli and pathological processes (21). However, the protective function associated with $\mathrm{HO}-1$ does not discriminate between cancerous and healthy cells; therefore, $\mathrm{HO}-1$ expression and activity may be associated with the growth, apoptosis, angiogenesis and metastasis of solid tumors (22). As a result, chemotherapy and radiotherapy may induce tumor resistance by promoting HO-1 expression in tumor cells, which consequently protects the tumor tissue. In addition, the protective effects of HO-1 on apoptosis are associated with the inhibition of the ROS generation (23); thus, in the present study, the interrelation between PEITC-induced apoptosis and HO-1 expression was investigated in K562 cells.

To verify that HO-1 was essential for K562 cell survival and ROS generation, the expression of HO-1 was regulated by treating the cells with hemin and ZnPPIX. Following stimulation with $20 \mu \mathrm{mol} / 1$ hemin and $10 \mu \mathrm{mol} / 1 \mathrm{ZnPPIX}$ for $48 \mathrm{~h}$, K562 cells were more prone to apoptosis, as suggested by the mRNA and protein analyses of cytochrome $c$, caspase-3, -9 and -8 , Fas and FasL. These apoptosis-related genes were found to exhibit the highest expression in the PEITC plus
ZnPPIX group of cells, and the lowest in the PEITC plus hemin group. Furthermore, combining PEITC and the HO-1 inhibitor ZnPPIX increased ROS generation in K562 cells.

In conclusion, PEITC may induce apoptosis by increasing caspase- $9,-8$ and -3 expression following the cytochrome $c$ release that occurs due to Fas- and FasL-mediated elevations in ROS production. Furthermore, the role of HO-1 in PEITC-induced apoptosis may provide novel insight for the treatment of CML.

\section{Acknowledgements}

This study was supported by grants from the National Natural Science Foundation of China (nos. 81270636 and 81070444).

\section{References}

1. Melo JV and Barnes DJ: Chronic myeloid leukaemia as a model of disease evolution in human cancer. Nat Rev Cancer 7: 441-453, 2007.

2. Conaway CC, Yang YM and Chung FL: Isothiocyanates as cancer chemopreventive agents: their biological activities and metabolism in rodents and humans. Curr Drug Metab 3: 233-255, 2002.

3. Sakao K, Desineni S, Hahm ER and Singh SV: Phenethyl isothiocyanate suppresses inhibitor of apoptosis family protein expression in prostate cancer cells in culture and in vivo. Prostate 72: 1104-1116, 2012.

4. Sarkars R, Mukherjee S and Roy M: Targeting heat shock proteins by phenethyl isothiocyanate results in cell-cycle arrest and apoptosis of human breast cancer cells. Nutr Cancer 65: 480-493, 2013.

5. Chan DK and Miskimins WK: Metformin and phenethyl isothiocyanate combined treatment in vitro is cytotoxic to ovarian cancer cultures. J Ovarian Res 5: 19, 2012.

6. Huong LD, Shin JA, Choi ES, et al: $\beta$-Phenethyl isothiocyanate induces death receptor 5 to induce apoptosis in human oral cancer cells via p38. Oral Dis 18: 513-519, 2012.

7. Yan H, Zhu Y, Liu B, et al: Mitogen-activated protein kinase mediates the apoptosis of highly metastatic human non-small cell lung cancer cells induced by isothiocyanates. Br J Nutr 106: 1779-1791, 2011.

8. Zhang Y: Cancer-preventive isothiocyanates: measurement of human exposure and mechanism of action. Mutat Res 555: 173-190, 2004

9. Huong le D, Shim JH, Choi KH, et al: Effect of $\beta$-phenylethyl isothiocyanate from cruciferous vegetables on growth inhibition and apoptosis of cervical cancer cells through the induction of death receptors 4 and 5. J Agric Food Chem 59: 8124-8131, 2011.

10. Kang L and Wang ZY: Breast cancer cell growth inhibition by phenethyl isothiocyanate is associated with down-regulation of oestrogen receptor-alpha36. J Cell Mol Med 14: 1485-1493, 2010.

11. Trachootham D, Zhou Y, Zhang H, et al: Selective killing of oncogenically transformed cells through a ROS-mediated mechanism by beta-phenylethyl isothiocyanate. Cancer Cell 10: 241-252, 2006.

12. Zhang H, Trachootham D, Lu W, et al: Effective killing of Gleevec-resistant CML cells with T315I mutation by a natural compound PEITC through redox-mediated mechanism. Leukemia 22: 1191-1199, 2008.

13. Miao RZ, Liu LQ, Chen L, et al: Activity of heme oxygenase-1 affects expression levels of hypoxia inducible factor-1 gene in vitro. Chin Med J (Engl) 125: 1310-1315, 2012.

14. Liao YF, Zhu W, Li DP and Zhu X: Heme oxygenase-1 and gut ischemia/reperfusion injury: A short review. World $\mathrm{J}$ Gastroenterol 19: 3555-3561, 2013.

15. Kobayashi T, Sato Y, Yamamoto S, et al: Augmentation of heme oxygenase-1 expression in the graft immediately after implantation in adult living-donor liver transplantation. Transplantation 79: 977-980, 2005.

16. Tibullo D, Barbagallo I, Giallongo C, et al: Nuclear translocation of heme oxygenase- 1 confers resistance to imatinib in chronic myeloid leukemia cells. Curr Pharm Des 19: 2765-2770, 2013. 
17. Chen C, Wang JS, Qin D, Yang Y, Yu YY and Fang Q: The effect of retrovirus-mediated HO-1 gene on chronic myeloid leukemia resistance cell K562/A02 apoptosis induced by nilotinib. Zhonghua Xue Ye Xue Za Zhi 33: 383-387, 2012 (In Chinese).

18. Zhang R, Humphreys I, Sahu RP, Shi Y and Srivastava SK In vitro and in vivo induction of apoptosis by capsaicin in pancreatic cancer cells is mediated through ROS generation and mitochondrial death pathway. Apoptosis 13: 1465-1478, 2008.

19. Wu CL, Huang AC, Yang JS, et al: Benzyl isothiocyanate (BITC) and phenethyl isothiocyanate (PEITC)-mediated generation of reactive oxygen species causes cell cycle arrest and induces apoptosis via activation of caspase-3, mitochondria dysfunction and nitricoxide (NO) in human osteogenic sarcoma U-2 OS cells. J Orthop Res 29: 1199-1209, 2011.
20. Cheung KL and Kong AN: Molecular targets of dietary phenethyl isothiocyanate and sulforaphane for cancer chemoprevention. AAPS J 12: 87-97, 2010

21. Hull TD, Bolisetty S, DeAlmeida AC, et al: Heme oxygenase-1 expression protects the heart from acute injury caused by inducible Cre recombinase. Lab Invest 93: 868-879, 2013.

22. Miyake M, Fujimoto K, Anai S, et al: Heme oxygenase-1 promotes angiogenesis in urothelial carcinoma of the urinary bladder. Oncol Rep 25: 653-660, 2011.

23. Calabrese V, Butterfield DA, Scapagnini G, Stella AM and Maines MD: Redox regulation of heat shock protein expression by signaling involving nitric oxide and carbon monoxide: relevance to brain aging, neurodegenerative disorders, and longevity. Antioxid Redox Signal 8: 444-477, 2006. 